$\begin{array}{ll}\text { le portiQue } & \text { Le Portique } \\ \text { Revue de philosophie et de sciences humaines }\end{array}$

$20 \mid 2007$

Gilles Deleuze et Félix Guattari : Territoires et devenirs

\title{
Le texte est le devenir de l'auteur/e
}

\section{Hélène Fau}

\section{OpenEdition}

\section{Journals}

\section{Édition électronique}

URL : http://journals.openedition.org/leportique/1371

DOI : 10.4000/leportique.1371

ISSN : $1777-5280$

\section{Éditeur}

Association "Les Amis du Portique"

Édition imprimée

Date de publication : 15 décembre 2007

ISSN : 1283-8594

\section{Référence électronique}

Hélène Fau, "Le texte est le devenir de l'auteur/e », Le Portique [En ligne], 20 | 2007, mis en ligne le 07 novembre 2009, consulté le 25 mars 2021. URL : http://journals.openedition.org/leportique/1371 : DOI : https://doi.org/10.4000/leportique.1371

Ce document a été généré automatiquement le 25 mars 2021

Tous droits réservés 


\title{
Le texte est le devenir de l'auteur/e
}

\author{
Hélène Fau
}

\author{
« To avoid discovery I stay on the run. To \\ discover things for myself I stay on the run ", \\ Jeanette Winterson, The PowerBook (3).
}

1 Il n'est pas rare que les études littéraires, en particulier lorsqu'elles s'intéressent aux courants postmodernes, qualifient les textes sur lesquels elles se penchent de textes « en devenir », désignant par ce biais des textes en mutation constante qui refusent toute fixité et ne cessent de bifurquer, au sens où Borges l'entendait. Mais l'antériorité de filiation dont l'auteur bénéficie - en tant que géniteur apparent du texte - ne laisset-elle pas supposer qu'il serait lui-même " en devenir » latent avant de placer ses écrits « en devenir » manifeste et qu'il activerait son « en devenir » à travers eux, anticipant ainsi inconsciemment, par et dans l'écriture, un avenir qui bien sûr resterait réellement et physiquement non vécu. Si tel était le cas, le texte serait alors une sorte d'horoscope, le parcours d'une vie, d'un destin qui aurait pu être vécu à la place de, une fenêtre sur le possible d'un autre monde, un formidable instrument visionnaire qui ouvrirait les portes d'une éternelle formation, déformation et transformation intérieures, d'une éternelle construction. "Nous philosophes, c'est par nos personnages que nous devenons toujours autre chose et que nous renaissons jardin public ou zoo ${ }^{1}$. » Bref, le texte pourrait être la lunette par laquelle l'auteur se voit, "se vit", se projette, se liquéfie et se remodèle, un peu comme les personnages des peintures de Bacon en dégoulinement permanent que Deleuze a également superbement éclairés.

C'est dans ce contexte d'hypothèses que, à partir du roman Art and Lies (1994) de Jeanette Winterson ${ }^{2}$, auteure britannique contemporaine, cet article propose, tout d'abord, d'expliquer ce que l'« en devenir » du texte, ou le texte en devenir, signifie, ce qu'il est et par quoi il se traduit, puis, à partir du roman de Virginia Woolf, la Promenade au phare (1927), de montrer brièvement ce à quoi l'« en devenir » de l'auteure, ou le devenir de l'auteure, peut correspondre. Le devenir refusant tout figeage, aussi bien substantiel, spatial que temporel, j'ai délibérément choisi de révéler l'un et l'autre dans deux romans différents, conçus à deux périodes différentes - moderne et postmoderne. Ce choix n'impose rien de définitif, tout roman pouvant contenir l'un et l'autre, l'un ou 
l'autre, ou ne contenir ni l'un ni l'autre. Bref, je me contenterai ici de poser un « problème » que je n'ai nullement l'ambition de résoudre.

\section{Pseudo-simultanéité et « en devenir » du texte}

3 La notion deleuzienne du devenir, «l'en devenir », est une notion particulièrement importante en littérature, car le texte littéraire, objet de bien des études, non seulement est mais devient. Il devient lorsqu'il est, il est lorsqu'il devient. Il est et devient en même temps. Il y a simultanéité. Mais cette simultanéité n'est pas une simultanéité de l'écriture, car il y a toujours un décalage entre l'écriture de l'un et l'écriture de l'autre, que ce soit au niveau du moment de l'écriture, de la forme qu'elle prend ou du fond qu'elle expose. Même l'« écriture collective ", telle que définie par Deleuze et Guattari, est une écriture plus consécutive, plus rhizomique que simultanée. Aussi la simultanéité, au sens auquel nous l'entendons, est-elle une simultanéité d'états et de passages à l'intérieur du cadre de représentation de l'écriture. Si nous prenons l'exemple d'un état A qui rejoint un état B par l'intermédiaire d'un passage 1 , nous constatons qu'il peut $\mathrm{y}$ avoir simultanéité à la fois entre $\mathrm{A}$ et 1 , entre 1 et $\mathrm{B}$ et entre $\mathrm{A}$ et B. Il convient, à ce stade, de rappeler le caractère musical de toute simultanéité, la musique étant l'une des seules vraies sphères de performance simultanée (possibilité de chanter, danser ou jouer un même morceau dans le même espace et le même temps). Reste cependant à savoir comment le texte littéraire entre dans une telle simultanéité. Il semble que ce soit l'introduction de paramètres langagiers tels que la métaphore (de l'image à l'idée), le palimpseste générique (du genre au genre) ou encore l'intertextualité (du texte au texte) qui en soit responsable, c'est-à-dire la superposition de deux matières en circulation osmotique l'une avec l'autre. Mais cette simultanéité littéraire à caractère musical reste relative. Elle n'est en rien absolue, car elle n'est qu'à caractère musical. Elle n'est pas musique. Ce n'est, en fait, qu'une pseudo-simultanéité, car l'inévitable écoulement du temps, même s'il est minime, altère - dans le moment même de la superposition - ce qui semble s'unir et veille ainsi à l'éternel maintien en mouvement des matières textuelles. L'élément « pseudo » est ici une chance, et non une entrave, car si la simultanéité de la performance textuelle était absolue, absolument musicale, il y aurait immobilité et le passage serait tout aussi figé que l'état dont il part. La métaphore, l'intertextualité, le palimpseste générique seraient alors choses mortes. Heureusement, il y a toujours un laps X de $\mathrm{A}$ à 1 , de 1 à $\mathrm{B}$ et/ou de $\mathrm{A}$ à $\mathrm{B}$ et c'est ce lapslà qui produit le mouvement et c'est ce mouvement-là qui, dans l'action littéraire, enclenche le véritable devenir du texte. Le mouvement n'est pas, contrairement aux apparences, le passage en soi, mais le passage alimenté ou porté par le moteur du temps, aussi minime soit-il. Le mouvement de l'espace textuel dépend du facteur temps. Et cela parce que la simultanéité n'est pas entière. Mais encore faut-il que le mouvement soit perçu. Rappelons à ce sujet la théorie de Roger Scruton ${ }^{3}$ qui dit que la musique, qu'il divise en trois domaines (le rythme, la mélodie et l'harmonie), ne prend forme que grâce à la représentation imaginaire que nous avons de l'espace et, par extension, du temps. Quand nous écoutons une mélodie, nous entendons un mouvement ou, plus exactement, croyons percevoir la mise en place d'un mouvement. Sans le transfert imaginatif de la notion d'espace, et de temps, le son perçu ne pourrait devenir note. Si nous appliquons cette même théorie au texte, nous obtenons : quand nous lisons un mot, une phrase, nous entendons un son et voyons donc un mouvement, 
du moins croyons-nous percevoir la mise en place d'un mouvement qui, invisiblement, fait avancer le texte.

\section{Métaphore-métamorphose}

4 Nous retrouvons cette représentation imaginative, cette chaîne associative entre le son que j'entends et le mouvement que je crois voir, dans le mécanisme métaphorique. En effet, de même qu'il y a, dans toute lecture ou dans toute écoute, transfert quasi simultané du son à l'idée d'espace et de temps, il y a, dans la métaphore, transfert quasi simultané de l'image à l'idée. Nous lisons une métaphore, entendons un son, des sons, voyons un mouvement d'image et récoltons une idée. La métaphore est ce que j'appellerai un oscillateur langagier, un instrument d'écriture servant à la mise en mouvement, à la mise "en devenir» du texte. Bien sûr, ce n'est pas le seul: le palimpseste générique, l'intertextualité ou encore la mise en abîme sont, eux aussi, des pendules, des balanciers qui, poussés par le temps, par le laps X, lancent et relancent le texte dans le mouvement, le propulsant ainsi, contre son gré, dans son « en devenir ».

5 Le roman Art and Lies de Jeanette Winterson est un roman à deux formes : un texte de 206 pages et un extrait de partition musicale de 9 pages (tiré de l'opéra Le Chevalier à la Rose de Richard Strauss). Ce qui semble être, à première vue, une juxtaposition, une consécutivité, est, en fait, un « en devenir » quasi simultané. Le texte devient, dès le début de sa narration, musique, sans cesser d'être texte. La partition y est invisiblement présente de même que le texte wintersonien reste invisiblement présent dans la partition au-delà de son dernier mot. Il enjambe la fin pour se poursuivre sous une autre forme. Il change tout simplement d'habit. Si, dans ce roman, nous prenons l'exemple de la métaphore, et d'une métaphore précise, à savoir la métaphore du train, nous constatons que celle-ci garantit un transfert doublement double étant donné que, d'une part, il y a passage de l'image à l'idée et de l'idée à l'image et que, d'autre part, ce passage est associé à un moyen de locomotion, c'est-à-dire de mouvement, le train, qui assure le transfert géographique de ville à ville, de pays à pays, et permet ainsi une transgression, à savoir la transgression de frontières qui, dans un premier temps, sont d'ordre physique. Dans le roman, le train traverse le paysage et la vie de l'un de ses passagers, «Handel » (clin d'œil à la musique). Mais notons que Handel est également un nom de passage à l'acte: "Handel», en allemand, signifie commerce, c'est-à-dire transfert de biens de main à main, le verbe "handeln» signifie "passer à l'acte, négocier"; l'anglais "to handle» indique, pour sa part, une manipulation ${ }^{4}$. Il caractérise la main qui travaille, qui passe à l'acte. Bref dans « handeln » et « to handle », il y a "hand", la main. Le nom de Handel est donc un nom chargé de passage, de mouvement, de manipulation, ce qui, bien évidemment, n'est pas sans importance dans le contexte dans lequel nous nous situons.

6 Handel est donc, de par son nom, personnage de passage et, de par son activité immédiate dans le roman, passager d'un train. Ce qu'il faut également mentionner à ce stade est que Handel, enfant, avait pour habitude de jouer avec un train miniature. Un jouet auquel l'enfant donne vie, mais que la vie abandonne un soir, le soir où l'enfantjoueur subit un abus sexuel. Le train-jouet est alors qualifié de train mort, «the dead train» (192). Et il faut savoir aussi que c'est par une main que l'abus sexuel passe, par la main du Cardinal Rosso, un religieux épris d'opéras, par la main donc d'un personnage fortement musical. Cette main, sa main, agit sur le corps de l'enfant Handel et c'est par 
cette main que la frontière de l'interdit est franchie. Aussi la main est-elle, au même titre que le train, un instrument de transgression. C'est par elle que Handel se verra condamné au rôle de castrat - la musique lui sera alors imposée - (pour ensuite devenir prêtre, puis chirurgien amateur de musique, le chirurgien étant celui qui opère par la main à corps ouvert). Aussi l'homme-passage, passager du train grandeur nature, a-t-il une vie aux dimensions infinies. Et changeantes.

7 La révélation du train miniature de l'enfant est de première importance, car c'est elle qui déclenche la compréhension du mécanisme métaphorique dans le roman. Elle est le déclic, le mouvement que nous croyons voir, comparable à la représentation imaginaire de l'espace et du temps évoquée précédemment, et qui nous permet de saisir, de toucher, l'« en devenir » du texte. Elle ne se fait que dans les dernières pages du roman (14 pages avant la fin) et ce n'est qu'à ce moment-là que la quasi simultanéité de l'oscillation est consommée, que l'idée est éclairée. C'est un peu comme si, à ce moment précis du déclic, l'image rassemblait, en accéléré, les mille plateaux qu'elle avait éparpillés dans le roman, à chaque entrée en scène du train, et allait, dans un élan spontané, cogner l'idée pour la remplir de substance. C'est dans cette pseudosimultanéité éclair de la métaphore du train que le texte est et devient - qu'il avance -, que le personnage est et devient - qu'il avance -, que le train est et devient - qu'il avance. De par ces mécanismes, texte et thématique avancent, collés l'un à l'autre. Nous sommes en plein flux. Nous sommes en pleins plis. Grâce à ce dépliage du mécanisme métaphorique, le lecteur comprend que le train réel, grandeur nature, dans lequel le personnage voyage, bouge et grandit et dont il ressortira métamorphosé, "déminiaturisé ", n'est pas un simple train, mais qu'il est le train du passage à la vraie vie, à l'âge adulte, le train dans lequel l'enfant mort vivant Handel monte et dont l'adulte vivant Handel descendra. Grâce à ce dépliage et déploiement, le lecteur comprend l'acheminement vers la musique par choix et non plus par obligation. Acheminement du personnage et du texte qui, ce faisant, se dirige tout droit vers la partition musicale de la fin. Le personnage, Handel, n'est plus le castrat qu'il était devenu contre son gré, enfant, mais un amoureux de la musique au nom de musique, Haendel. En fait, au niveau thématique, l'« en devenir" correspond à une « déminiaturisation » de l'objet, le train, et du personnage, Handel, dans la traversée de l'espace (mental et géographique) et, au niveau textuel, à une « démétaphorisation » de la métaphore, car, en révélant son mécanisme, le texte la « littéralise ", la met à nu ou, en d'autres termes, la "défonctionnalise " une fois sa fonction remplie. La métaphore est en quelque sorte relâchée après avoir propulsé le texte un peu plus loin dans son " en devenir ». Ce qui est par ailleurs confirmé par une re-définition de la métaphore que nous livre Sappho, autre personnage du roman. Et ce n'est sûrement pas un hasard si c'est Sappho l'écrivaine, celle qui détient et manipule le mot par la main de l'écriture, celle par laquelle le Verbe se forme et passe, celle dont le nom sans cesse renouvelé est passé d'histoire en histoire, de siècle en siècle, qui se fait le porteur de cette nouvelle définition: "What is remembered is not a deed in stone but a metaphor. Meta = above. Pherein = to carry. That which is carried above the literalness of life. A way of thinking that avoids the problems of gravity » (136-137). La métaphore est donc ce qui flotte au-dessus de nos têtes, de nos vies, ce qui lève le poids et permet au texte et au personnage d'avancer, de bouger, de devenir. Cette nouvelle définition est donnée à la page 136-137, c'est-à-dire 55 pages avant la révélation du mécanisme métaphorique, ce qui prouve qu'elle agit en précurseur, en héraut, en messager-annonceur. 
8 La métaphore du texte wintersonien est une métamorphose. Et dans pareil cas, il n'y a aucune raison de préférer l'une à l'autre. Au même titre que les autres oscillateurs langagiers, la métaphore conduit le texte à l'embouchure de sa perpétuelle renaissance, un texte qui - dans le roman de Winterson - se jette visiblement et concrètement dans l'avenir d'un opéra passé. Il place la musique du passé dans l'ouverture de l'aprèstextuel. Il s'agit d'un acte rétro-prospectif. L'opéra devient, par ce biais, ouverture, passage vers l'au-delà, et non fermeture de ce qui précède. Il faut préciser que la partition musicale ne fait que prêter un visage à l'« en devenir ». Un parmi d'autres. Mais ce visage est absolument nécessaire, car il permet de visualiser l' " en devenir » du texte au moment de la lecture. Bref, il faut comprendre cette oscillation en simultané comme une métamorphose, une ouverture sur le neuf, le neutre, le "toujours déjà » "en devenir", le rétro-prospectif, comme ce qui fut et qui s'achemine vers une nouvelle (re)composition. La (re)composition n'étant pas un retour à ce que la composition fut, mais la composition de la composition, c'est-à-dire une avancée dans la composition même (pour reprendre les termes de Vladimir Jankélévitch à propos de la reconnaissance (connaissance de la connaissance). Il faut la comprendre comme une ouverture sur le vrai, comme un abandon du mensonge. Art and Lies se muerait alors en Art and Truth. Mais, dans la fiction wintersonienne, tout est, ne l'oublions pas, sans vraiment être. Tout est placé sous le signe de la non fixité. Notons que la partition reste par ailleurs en suspens. L'opéra de Richard Strauss compte en fait un acte de plus, intitulé «Ist ein Traum, kann nicht wirklich sein... » (C'est un rêve, ça ne peut réellement exister). Mais le point d'interrogation posé par l'illusion du rêve n'est-il pas, lui aussi, un « en devenir »?

\section{Géométrisation et « en devenir » de l'auteur/e}

9 La question qu'il convient peut-être de se poser à ce stade est : l'« en devenir » du texte agit-il en autonomie ou intervient-il en tant que masque? Serait-il un prétexte, une manière détournée d'exprimer l'« en devenir » du tisserand de l'histoire ? Voyons ce qu'il en est, peut-être, dans la Promenade au phare de Virginia Woolf. To the Lighthouse, en anglais ${ }^{5}$. Le roman se compose de trois parties inégales : la $1^{\text {ère }}$ très longue (186 pages sur 306 au total) se déroule sur un week-end, la $2^{\mathrm{e}}$ très courte (seulement 25 pages) enjambe dix années et la $3^{\mathrm{e}}$ d'une longueur moyenne (93 pages) repose sur quelques jours. Texte et temps ${ }^{6}$ sont longs, puis courts, puis ni longs ni courts, ce qui signifie que nous assistons à une oscillation entre trois sphères de durée de représentation différente(s). Temps, espace et texte - temps et espace dans le texte co-tourbillonnent étroitement mêlés l'un à l'autre. Il y a pliage, dépliage, repli(age). Dans la longueur de la 1ère partie, véritable étalage des trompe-l'œil de l'existence, le personnage central, Mrs Ramsay, qui accueille différents invités dans sa superbe demeure le temps d'un week-end, joue les mères de famille comblées. Cependant, dans l'entre-deux des dialogues, le lecteur capte son insatisfaction et son inachèvement. Aussi son effacement physique, c'est-à-dire sa mort, brièvement évoquée dans la $2^{\mathrm{e}}$ partie, ne surprend-il pas. Il annule l'attachement à l'habitation familiale précédemment affiché. Il libère la figure architecturale - la maison ne sera jamais plus ce qu'elle a été jusqu'alors - et son habitant-clé. Il déterre les racines. Condition préalable à la survie, à la non suffocation. En effet, le lecteur attentif saisit rapidement que Mrs Ramsay est toujours parce que, en fait, elle n'a jamais cessé de devenir et 
qu'elle est devenue et devient par le biais de Lily Briscoe, jeune peintre invitée par la famille dans la 1ère partie, et avant tout par le biais du tableau de Lily Briscoe. Car Lily n'a d'autres préoccupations que de peindre le portrait de Mrs Ramsay, du début à la fin du roman, un portrait aux multiples formes - de l'arbre feuillu rond au simple trait plat - qui ne sera achevé que dans les toutes dernières lignes. En fait, et c'est là la révélation, l'achèvement du tableau se traduit par l'abandon de la figure, de la forme circulaire au profit du plateau, de l'arbre au profit de la ligne tracée au centre (306), un ensemble de points en flottement qui ne sont retenus ni par un début ni par une fin, un milieu " acentré » alliant un point quelconque à un point quelconque. Il s'agit ici d'une géométrisation de la figure qui signe la fin du tableau de Lily et la victoire du rhizome sur la racine. "La peinture", écrivait Gilles Deleuze, «doit arracher la figure au figuratif ${ }^{7}$ ». Un " devoir » auquel Lily Briscoe ne manque pas. Et dans l'étalage de son geste libérateur, nous comprenons que Mrs Ramsay devient enfin ce qu'elle a toujours voulu être et n'a jamais été. Elle quitte sa figure, ses formes, ses couleurs, ses racines enchevêtrées qu'elle n'avait pas choisies et entre dans l'abstrait pour atteindre l'inatteignable, le phare, libre et en flottement, lui-même « en devenir » sur des eaux " en devenir " permanent. Pli, dépli(age), repli des vagues. Deleuze écrivait à propos des romans de Melville qu'il n'y avait « qu'une seule Figure originale comme l'unique soleil d'une constellation d'univers, comme commencement des choses, ou comme le phare qui tire de l'ombre un univers caché ${ }^{8}$ ». Le phare, Mrs Ramsay, Lily, le tableau, le phare: tous unique figure, figure unique libérée par la défiguration de la géométrisation des dernières lignes, de LA ligne ${ }^{9}$. Ce qu'il importe de souligner ici est que l'« en devenir» de Mrs Ramsay, son dépassement en formation perpétuelle, ressemble à un suicide surmonté, au suicide anticipé et surmonté de Virginia Woolf. Du moins, d'après le regard anachronique que nous lui portons, pourrait-il être compris de la sorte. L'auteure Woolf est, existe, par Mrs Ramsay et devient autre par Lily et son tableau même si elle ne se confond, à aucun moment, avec l'une ou avec l'autre. Auteur et personnage, au même titre qu'être et devenir, sont deux évolutions " aparallèles ». L'un et l'autre évoluent dans d'infinies dimensions mouvantes, l'un et l'autre étant parfois l'un, parfois l'autre, parfois ni l'un ni l'autre, parfois autre que l'un ou l'autre. Le tableau n'est, quant à lui, qu'un plan d'immanence aux infinis plans. C'est un peu comme si l'auteure surmontait et survivait à son suicide avant même de l'avoir commis, comme si elle le commettait par écrit avant de le commettre en nature - quatorze ans plus tard - tout en sachant qu'elle y survivrait par le tracé du trait de Lily Briscoe qui est une fin sans en être une, comme si le roman, par l'intermédiaire du tableau, était une simulation, une répétition générale de ce qui adviendrait ultérieurement. Ce n'est pas au fond de l'eau que l'auteure Woolf se dirigerait, mais à sa surface qu'elle choisirait de flotter avant d'atteindre les hauteurs du phare. "Écrire", disait Deleuze dans l'Abécédaire, "c'est témoigner de la vie, pour la vie. " La diégèse et sa représentation textuelle seraient ainsi "nomadisées» par l'auteure au profit de son propre «en devenir ». Elles sont son devenir vrai, le dépassement de sa mélancolie, " la troisième personne de son "Je" ", "l'envers de sa carte». Elles se font ici «au nom d'un dehors ${ }^{10} »$, au nom du phare.

10 Le plus effarant est que, à ce stade de l'analyse, la géométrisation de la figure, telle que pratiquée dans le roman woolfien, semble renvoyer au texte en devenir (musical) de Art and Lies. Il y a « aplanissement » de la figure dans l'un comme dans l'autre et c'est dans cet "aplanissement» que l'un et l'autre correspondent dans une invisibilité sans précédent. En effet, que sont les notes et portées de l'extrait de partition du Chevalier à 
La Rose de Strauss, livrées en fin de roman, si ce n'est une reproduction graphique minimaliste de l'idée qu'elles expriment. Ne schématisent-elles pas au même titre que la ligne du tableau de Lily Briscoe? C'est Joachim Dupuis qui, dans un article fort intéressant, rappelle l'origine de la note et de la ligne musicale. "L'auteur [compositeur] ", écrit-il, « procède à l'élaboration graphique de figures (avec les notes et les lignes de la partition) : il y a géométrisation du motif, un modelage graphique perpétuel, comme s'il s'agissait d'écrire à partir d'un alphabet réduit qu'on ferait moduler pour en rendre le maximum de sons et de figures ${ }^{11}$ ». Certes, Dupuis semble vouloir souligner la construction de figures, à partir d'éléments graphiques, - un peu comme un Golem émergeant de la glaise -, et non la sortie du figuratif par le recours à la géométrisation. Mais la construction de figures sur laquelle il attire notre attention ne serait-elle pas ce qui (re)viendrait dans l'après-géométrisation? Il est clair que le processus de géométrisation est tout aussi flottant, tout aussi «en devenir » que la figure qu'il défigure et que, cela étant, il aura une suite. Mais il est également indéniable que, s'il y a post-géométrisation, il y a eu pré-géométrisation et que cette pré-géométrisation, sous-jacente dans la réflexion de J. Dupuis, n'est autre que l'ère primaire du figuratif. Un figuratif peuplé de figures et occupé par l'Idea, point de départ de toute composition. Bref, dans un premier temps, "le modelage graphique " ne défigurerait, ne styliserait, ne simplifierait que la Ur-figure dont il s'inspirerait et ce ne serait que dans un deuxième temps qu'il déboucherait, sans doute, sur la construction d'un nouveau figuratif (peut-être imaginaire ?). C'est à cette étape-là que J. Dupuis fait référence. Et c'est cette géométrisation de la Ur-figure, personnage et/ou texte, qui agit en trait d'union entre la Promenade au phare et Art and Lies. Un trait d'union entre deux traits : entre le trait du tableau et le trait de la portée musicale. Des traits qui, par ailleurs, ne sont pas sans rappeler le graphisme utilitaire et le profil épuré chéris par le Bauhaus, mouvement artistique des années 1920 qui favorise l'abstrait des formes géométriques au détriment des figures d'un impressionnisme social et d'un expressionnisme chargé d'émotions et d'intellectualisme. Des traits, cubes, triangles et autres qui s'imposent en résonances musicales et rythmiques. Un peu comme dans les tableaux de Paul Klee dans lesquels, sur lesquels, desquels la ville imaginaire de Beribe surgit sous l'apparence de traits rappelant les notes et les lignes de la portée musicale (Beribe, Wasserstadt ; Beribe, Universität [1927]). Virginia Woolf, ses collègues modernes et postmodernes à venir, ne seraient-ils pas, eux aussi, de cette lignée? N'oublions pas que c'est en 1927 que le roman la Promenade au phare est publié. En pleine période Bauhaus. N'oublions pas que, soixante-dix ans plus tard, Jeanette Winterson se réclame de l'héritage woolfien. Des traits, symboles d'un désir de transformation, d'une sortie du figuratif, d'un devenir par le biais de l'art, aussi minimaliste soit-il. Des traits hors du temps et de l'espace. Car le devenir est sans domicile fixe et sans âge.

11 Il est enjambement, pour entrer dans une, deux, trois, mille dimensions de réalités toutes beaucoup plus réelles, bien qu'invisibles à l'œil nu, que celle que l'on est programmé à écrire, à lire, à vivre et à quitter. Le corps passe, mais le texte (son histoire et ses mécanismes) reste - en mouvement -, et l'auteur/e ne cesse jamais de devenir à travers eux. Le trait du milieu tracé par Lily Briscoe et le trait de la note retracée par Jeanette Winterson ne sont pas une fin mais un départ toujours déjà relancé. Des lignes de fuite sans points de chute. 


\section{NOTES}

1. Gilles DELEUZE, Qu'est-ce que la philosophie ?, Paris, Éditions de Minuit, 1991, p. 71.

2. . Jeanette WINTERSON, Art and Lies, 1994, London, Vintage International, 1996.

3. . Roger SCRUTON, The Aesthetics of Music, Oxford, Clarendon Press, 1997, du moins l'un des volets théoriques développés dans l'ouvrage et analysé par Malcolm BUDD dans l'article «Musical Movements and Aesthetic Metaphors " paru dans The British Journal of Aesthetics 43 en été 2003, p. 209-223, ici p. 210.

4. . Renvoi à L'Amour du loup et autres remords (Die Ursache - La Chose) dans lequel Hélène Cixous rappelle l'intervention de la main dans toute forme de passage en faisant référence aux verbes anglais, « to handle », et allemand, « handeln », p. 123. Voir Hélène cIxous, L'Amour du loup et autres remords, Paris, Galilée, 2003.

5. . Virginia woolf, To the Lighthouse, 1927, London, Penguin, 1996.

6. . Temps à la fois intérieur et extérieur à l'histoire (homo- et hétérodiégétique) : d'une part, le week-end vécu (par les personnages) et partagé (par le lecteur) dans le premier chapitre et, d'autre part, les dix années qui se sont écoulées hors texte entre le 1er et le 2e chapitre.

7. . Sur la sortie du figuratif, voir Gilles Deleuze, Francis Bacon. Logique de la sensation, Paris, Seuil, 2002 [1981], ici p. 17.

8. . Gilles Deleuzeet Félix GUATTARI, Qu'est-ce que la philosophie ?, Paris, Éditions de Minuit, 1991, p. 64.

9. . La géométrisation, ici évoquée, fait l'objet d'une autre étude, placée sous le signe de la déterritorialisation deleuzienne et qui sera publiée dans l'un des prochains numéros du Portique.

10. . Gilles deleuzeet Félix GuATtARI, Mille Plateaux, Paris, Éditions de Minuit, 1980, p. 34.

11. . Joachim DupuIs, "Variations, métamorphoses et cristal à propos de la rencontre entre musique et pensée dans le minimalisme », www.musicologie.org, 2004, p. 4.

\section{RÉSUMÉS}

La notion deleuzienne du « devenir » agit aussi bien sur le texte littéraire que sur son auteur/e. Elle invite à se demander si l'apparent géniteur ne serait pas « en devenir » latent avant de placer ses écrits « en devenir » manifeste et à s'interroger sur les pouvoirs visionnaires de sa production lettrée qui déboucherait alors sur une éventuelle éternelle (re)construction. C'est dans une pseudo-simultanéité d'états et de passages, métaphoriques et autres, à caractère musical que le texte littéraire se mue, avance et devient, qu'il est peu à peu "nomadisé » au profit de "l'en devenir » de l'auteur/e. C'est ce que le présent article tente de révéler, à partir de deux romans, l'un postmoderne - Art and Lies (1994) de Jeanette Winterson - et l'autre moderne - To the Lighthouse (1927) de Virginia Woolf.

The text serves the author's own becoming The deleuzian concept of "devenir" interacts with the literary text as well as with its author. The author might be invisibly (or unconsciously) "en devenir" before visibly (or consciously) releasing his/her writings into an always about-tobecome sphere of representation. Could the literary production be thus seen as a coded visionary path, always on the move, showing us what might be - at both textual and authorial levels - and 
possibly leading to an eternal (re)construction (of the text and of its creator)? The literary text seems to move and become through and within pseudo-simultaneous performances, reminding of musical devices. It seems to be progressively made "nomadic" in order to serve the author's own becoming. The present article offers to explore both levels within two novels written at two different periods, Art and Lies (1994), Jeanette Winterson and To The Lighthouse, Virginia Woolf (1927).

Werden durch und mit dem Text Es sieht aus, als ob das Konzept des „devenir“, so wie Gilles Deleuze es definiert, sowohl auf der Text - als auch auf der Autorenebene wirken könnte. Der literarische Text bewegt sich innerhalb pseudo-simultaner Zustände, metaphorischer und musikalischer Art, und ist dadurch permanent am Werden. Diese immer wieder kehrende Mutation geschieht zugunsten des „devenir" des Autors. Die Analyse wird anhand von zwei Romanen - To the Lighthouse von Virginia Woolf und Art and Lies von Jeanette Winterson durchgeführt.

\section{AUTEUR}

\section{HÉLÈNE FAU}

Hélène Fau est Docteure en littérature anglaise de l'Université Paul Verlaine de Metz. Titre de thèse (soutenue en 2003) : Mouvements baroques et néo-baroques dans l'œuvre romanesque de Jeanette Winterson : entrée dans l'au-delà du postmodernisme, Publications du CETT, Série 2005, $\mathrm{n}^{\circ}$ 8. Elle travaille actuellement comme enseignante (niveau MCF) au Département de français de l'Université de la Sarre (Sarrebruck, Allemagne). Membre associé du Centre de Recherche Écritures (Axe 4) de l'Université Paul Verlaine de Metz. 